\title{
A Study of Inducible Clindamycin Resistance among Staphylococcus aureus Skin and Soft Tissue Infections in A Tertiary Care Hospital
}

\author{
Abirami Lakshmy Jayachandran ${ }^{1}$, Balan Kandasamy ${ }^{1}$, Sangeetha Vilwanathan ${ }^{2}$, Sheila Doris Devamani \\ T. $^{1}$, Vanitha Devi E. ${ }^{1}$, Vandhitha Muralidharan ${ }^{1}$ \\ ${ }^{1}$ Department of Microbiology, Karpaga Vinayaga Institute of Medical Sciences and Research Centre, Madurantakam, \\ Tamilnadu, India \\ ${ }^{2}$ Department of Microbiology, Central Leprosy Training and Research Institute, Chengalpet, Tamilnadu, India
}

\begin{abstract}
Objectives: Drug resistant phenotypes like MRSA are difficult to treat requiring higher group of antibiotics. Topical agents like clindamycin can be used for the therapy of MRSA. The knowledge of prevalence of inducible clindamycin resistance phenotype is essential to prevent treatment failure.

Methods: A total of 204 staphylococcal isolates obtained from skin and soft tissue infections and MRSA was detected by Cefoxitin disc diffusion method and detection of $M e c A$ gene by Polymerase chain reaction (PCR). Antibiotic susceptibility testing was performed by Kirby Baeur disc diffusion method. The Erythromycin resistant isolates were tested for D test. The differences in antibiotic susceptibility pattern between MRSA and MSSA was compared by Chi Square test using Graph pad Quick Calcs software and p value less than 0.05 was considered as significant
\end{abstract}

Results: Out of the 204 Staphylococcus aureus isolates, 48 (23.5\%) were identified as MRSA by Cefoxitin disc diffusion method. All these $48(23.5 \%)$ isolates were also positive for $\mathrm{Mec} A$ gene by PCR. Inducible clindamycin resistance (iMLSB resistance phenotype) was observed among $24(11.7 \%)$ of the isolates. MRSA showed comparatively lesser susceptibility than MSSA $(p \leq 0.05)$. Among the MRSA inducible clindamycin resistance was seen among 11 (22.9\%).

Conclusions: Emergence of drug resistance warrants antibiotic susceptibility testing for all the isolates in the laboratory. Cefoxitin disc diffusion method can be used in resource constraint laboratory where PCR facilities are not available. Inducible clindamycin resistance phenotype (iMLS phenotype) must be checked for all isolates showing erythromycin resistance to prevent treatment failure. J Microbiol Infect Dis 2019; 9(3):125-128.

Keywords: Methicillin resistant Staphylococcus aureus (MRSA), iMLSB resistance phenotype

\section{INTRODUCTION}

Infections caused by Staphylococcus aureus is a growing problem worldwide with Methicillin resistant Staphylococcus aureus (MRSA) posing a therapeutic challenge. The various risk factors associated with MRSA infections are previous hospitalizations [1]. Erythromycin and clindamycin are used as topical therapeutic agents for treatment of skin and soft tissue infections associated with Staphylococcal infections [2].

However wide spread use of the Macrolide, Lincosamide, Streptogramin group of drugs have led to emergence of resistance. The erm gene is responsible for clindamycin resistance. The knowledge of prevalence of inducible clindamycin resistance phenotype is essential to prevent treatment failure.

The present study aims to isolate and identify the MRSA by Cefoxitin disc diffusion method and Polymerase chain reaction for detection of $M e c A$ gene and to detect inducible clindamycin resistance by $D$ test. A positive $D$-test indicates the presence of an erm gene resulting in clinical failure [3].We also aim at ascertaining the difference in $D$ test among MRSA and Methicillin Sensitive Staphylococcus aureus (MSSA).

Correspondence: Dr. Abirami Lakshmy Jayachandran, Department of Microbiology, Karpagavinayaga Institute of Medical Sciences and Research Center, Madhurantakam, Kanchipuram, Tamilnadu, India 


\section{METHODS}

The present study was conducted from September 2016 to June 2017 at the department of microbiology and approved by the institutional ethical committee. A total of 204 Staphylococcus aureus isolates were obtained from skin and soft tissue infections and included in the study. The isolates were identified as Staphylococcus aureus by Standard Microbiological techniques.

Antibiotic susceptibility testing was done by Kirby Bauer's disc diffusion method using Amoxicillin $(30 \mu \mathrm{g})$, Erythromycin $(15 \mu \mathrm{g})$, Clindamycin $(2 \mu \mathrm{g})$, Gentamicin $(30 \mu \mathrm{g})$, Cotrimoxazole $(25 \mu \mathrm{g})$, Ciprofloxacin $(5 \mu \mathrm{g})$, Cefoxitin $(30 \mu \mathrm{g})$, Vancomycin $(30 \mu \mathrm{g})$ and linezolid $(30 \mu \mathrm{g})$.

Methicillin resistance was detected by Cefoxitin disc diffusion method and results interpreted as per CLSI guidelines [4].

The isolates showing Erythromycin resistance were subjected to $\mathrm{D}$ test. The results were interpreted as per CLSI guidelines [4].

The erythromycin disc $(15 \mu \mathrm{g})$ disc was placed at a distance of $15 \mathrm{~mm}$ edge to edge and incubated at $37^{\circ} \mathrm{C}$. The results were interpreted as per CLSI guidelines. Flattening of zone (iMLSB resistance phenotype, D shape near the clindamycin disc) indicates a positive test for inducible clindamycin resistance. Two other phenotypes was also be interpreted [5].MS phenotype- circular zone of inhibition around clindamycin disc and resistance to erythromycin, $\mathrm{CMLS}_{\mathrm{B}}$ phenotype- resistance to both clindamycin and erythromycin.

\section{Polymerase chain reaction for detection of Mec A gene}

HiPurATM Genomic purification kit (HIMEDIA) was used for DNA isolation and the steps were followed as per the manufacturer's instruction. MRSA Detection Kit (Uniplex) was utilized for amplification of Mec $A$ gene using specific primers and controls. The following PCR program was followed. Initial denaturation of 94 ${ }^{0} \mathrm{C}$ for 10 minutes following by denaturation at $94^{\circ} \mathrm{C}$ for 1 minute, Annealing at $60{ }^{\circ} \mathrm{C}$ for 1 minute followed by extension at $72{ }^{\circ} \mathrm{C}$ for 1 minute (30 cycles) and final extension at $72{ }^{\circ} \mathrm{C}$ for 10 minutes. After amplification the products were subjected to Agarose gel electrophoresis [6].

\section{Agarose gel electrophoresis}

The amplicons were loaded along with $6 \mathrm{X}$ gel loading dye on to $1.5 \%$ agarose gel incorporated with Ethidium bromide .DNA ladder (100 bp) was also loaded for confirming the size of the amplicon and observed under UV trans illuminator. For the isolates positive for Mec $A$ gene bands were observed at (293 bp) [6].

\section{Statistical Analysis}

The differences in antibiotic susceptibility pattern between MRSA and MSSA was compared by Chi Square test using Graph pad Quick Calcs software and $p$ value less than 0.05 was considered as significant.

\section{RESULTS}

A total of 204 Staphylococcus aureus isolates were obtained from skin and soft tissue infection over a period of one year. Among them 48 (23.5\%) were identified as MRSA by Cefoxitin disc diffusion method. All these strains were also positive for Mec $A$ gene by Polymerase chain reaction. Overall highest sensitivity was observed for Ciprofloxacin 164(80.34\%) and Gentamicin 156(76.47\%). Least susceptibility was observed for Amoxicillin 66(32.3\%).All the isolates were sensitive to Vancomycin and Linezolid.

In the present study MS phenotype (Erythromycin resistance) was seen among 63 $(31 \%)$ of the Staphylococcal isolates. $\mathrm{cMLS}_{\mathrm{B}}$ phenotype was observed among $38(18.6 \%)$ and Inducible clindamycin resistance was noted among $24(11.7 \%)$ of the isolates (Figure 1). Among the MRSA 11(22.9\%) showed inducible clindamycin resistance phenotype. There was a significant difference in the Antibiotic sensitivity pattern among the MRSA and MSSA (Methicillin sensitive Staphylococcus aureus) as depicted in Tablel 1 ( $p \leq 0.05)$. MRSA isolates showed lesser susceptibility than MSSA for all the antibiotics tested except for amoxicillin where MSSA showed a higher susceptibility. ( $p=0.543)$.

Among the 48 isolates of MRSA, 22 were of Community origin (CA- MRSA) and 4 (18.18\%) isolates showed inducible clindamycin resistance. 


\section{DISCUSSION}

Emergence of Multidrug drug resistant organism has made determining the antibiotic susceptibility pattern as essential. In the present study the Prevalence of MRSA was 23.5\%.Studies across India has reported prevalence of MRSA ranging from $23 \%$ to as high as $74 \%[7,8]$. These differences could be attributed to variations in risk factors for acquisition of resistance and infection prevention practices and antibiotic stewardship programs.

We have compared Cefoxitin disc diffusion method and PCR to detect Mec A gene for the identification of MRSA isolates. Both the methods were $100 \%$ in concordance with each other in the present study. All the isolates that were identified as MRSA by Cefoxitin disc diffusion method was also Mec $A$ gene positive by PCR similar to other studies[6]. Detection of Mec $A$ gene is the gold standard method for identification of MRSA. In case of resource constraint setups Cefoxitin a inducer of $M e c A$ gene can be used for detecting MRSA.

The lesser susceptibility was observed among the MRSA strains for erythromycin, Clindamycin, Gentamicin, Ciprofloxacin, Cotrimoxazole compared to MSSA as depicted in table 1 which is statistically significant $(p \leq 0.001)$. This was in concordance with other Studies who also reported that drug susceptibility was comparatively lower among the MRSA [9-14].An exception was Amoxicillin where the susceptibility was higher among the MSSA. Susceptibility of $100 \%$ was observed for
Vancomycin and Linezolid. Usage of these reserve drugs should be restricted only to MRSA. This will prevent the emergence of glycopeptide resistance. Since therapeutic options for multidrug resistant isolates like MRSA is limited. Many treatment options are available for Skin and soft tissue infections caused by Staphylococcus with clindamycin being used for treatment of both MSSA and MRSA infections. Emergence of inducible clindamycin resistance is on the rise. A study by Aleksandra $A D$ et al in central Serbia has reported iMLSB phenotype as high as 50\% among the Staphyloccocal isolates [15]. Shenoy MS et al have reported inducible clindamycin resistance as $15.65 \%$ in a study in south India[16].A study by Deotale $V$, et al have reported iMLSB phenotype resistance percentage in the range of $27.6 \%$ among MRSA isolates [5]. In our hospital the overall prevalence of inducible clindamycin resistance among Staphylococcal isolates was 24 (11.7\%) similar to Phukan $C$ et al [8] .It was also observed that among MRSA $22.9 \%$ of the isolates showed inducible Clindamycin resistance.

Reporting of isolates as susceptible to clindamycin without checking for inducible resistance phenotype (iMLS phenotype) will result in treatment failure. The raising resistance to Clindamycin is of important concern as isolates reported as sensitive without checking for inducible resistance by $D$ test on those isolates showing Erythromycin resistance will result in treatment failure.

Table 1. Clindamycin susceptibility patterns of the Staphylococcal isolates

\begin{tabular}{lccc}
\hline Antibiotics & MRSA, n (\%) & MSSA, n (\%) & P Value \\
\hline Erythromycin & $15(31.25 \%)$ & $126(80.76 \%)$ & $<0.001$ \\
Clindamycin & $16(33.33 \%)$ & $132(84.61 \%)$ & $<0.001$ \\
Gentamicin & $16(33.33 \%)$ & $140(89.74 \%)$ & $<0.001$ \\
Ciprofloxacin & $26(54.16 \%)$ & $138(88.46 \%)$ & $<0.001$ \\
Cotrimoxazole & $18(37.50 \%)$ & $116(74.35 \%)$ & $<0.001$ \\
Amoxicillin & $10(20.83 \%)$ & $56(35.89 \%)$ & 0.0543 \\
Vancomycin & $48(100 \%)$ & $156(100 \%)$ & NA \\
Linezolid & $48(100 \%)$ & $156(100 \%)$ & NA
\end{tabular}

MRSA=Methicillin-Resistant Staphylococcus aureus, MSSA= Methicillin-Sensitive Staphylococcus aureus

Number of $M R S A=48$, Number of $M S S A=156$ 
In conclusion Methicillin resistance should always be routinely identified in the laboratory as shown by higher antibiotic resistance among MRSA. Cefoxitin disc diffusion method should be used as method of detection of MRSA in resource constraint labs where $M e c A$ gene detection is not feasible. Effective Infection control practices and surveillance protocols can prevent MRSA associated infections. All erythromycin resistant Staphylococcal isolates should be tested for inducible clindamycin resistance to prevent treatment failure.

\section{ACKNOWLEDGMENTS}

Declaration of Conflicting Interests: The authors declare that they have no conflict of interest.

Funding: This project received no specific grant from any funding agency in the public, commercial, or not-for-profit sectors.

\section{REFERENCES}

1. Arora S, Devi P, Arora U, Devi B. Prevalence of Methicillin- resistant Staphylococcus aureus (MRSA) in a tertiary care hospital in northern India. J Lab Physicians 2010; 2(2):78-81.

2. Ajantha GS, Kulkarni RD, Shetty J, Shubhada C, Jain $P$. Phenotypic detection of inducible clindamycin resistance among Staphylococcus aureus isolates by using the lower limit of recommended inter-disk distance. Indian J Pathol Microbiol 2008; 51(3)376378.

3. Levin TP, Suh B, Axelrod P, Truant AL, Fekete T. Potential clindamycin resistance in clindamycinsusceptible, erythromycin-resistant Staphylococcus aureus: Report of a clinical failure. Antimicrob Agents Chemother 2005; 49:1222-1224 (13).

4. Clinical and laboratory standards institute. Performance standards for antimicrobial susceptibility testing.Clinical Laboratory Standards Institute.Wayne, PA, USA, M100S, $26^{\text {th }}$ edition 2016; 36:74-81.

5. Deotale V, Mendiratta DK, Raut U, Narang P. Inducible clindamycin resistance in Staphylococcus aureus isolated from clinical samples. Indian $\mathrm{J}$ Med Microbiol 2010; 28:124-126.

6. Anand KB, Agrawal P, Kumar S, Kapila K. Comparison of the Cefoxitin disc diffusion test, the oxacillin screen agar test and PCR for the Mec A gene for detection of MRSA. Indian J Med Microbiol 2009; 27: 27-29.

7. Prabhu K, Rao S, Rao V. Inducible clindamycin resistance in Staphylococcus aureus isolated from clinical samples. J Lab Physicians 2011; 3:25-27.
8. Phukan C, Ahmed GU, Sarma PP. Inducible clindamycin resistance among staphylococcus aureus isolates in a tertiary care hospital of Assam. Indian J Med Microbiol 2015; 33:456-458.

9. Vidhani S,Mehndiratta $P$, Mathur M. Study of methicillin resistant S.aurus (MRSA) isolates from high risk patients. Indian J Med Microbiol 2001;19:1316.

10. Menezes GA, Harish BN, Sujatha S, Vinothini K, Parija SC. Emergence of vancomycin-intermediate Staphylococcus species in southern India. J Med Microbiol 2008; 57:911-912.

11. Majumder D, Bordoloi JS, Phukan AC, Mahanta J. Antimicrobial susceptibility pattern among methicillin resistant staphylococcus isolates in Assam. Indian $\mathrm{J}$ Med Microbiol 2001; 19:138-140.

12. Indian Network for Surveillance of Antimicrobial Resistance INSAR) Group, India. Methicillin resistant Staphylococcus aureus (MRSA) in India: Prevalence and susceptibility pattern. Indian J Med Res 2013; 137:363-369.

13. Tiwari HK, Das AK, Sapkota D, Sivarajan K, Pahwa VK. Methicillin resistant Staphylococcus aureus: Prevalence and antibiogram in a tertiary care hospital in western Nepal. J Infect Dev Ctries 2003; 3:681-684.

14. Rajaduraipandi K, Mani KR, Panneerselvam K, Mani M, Bhaskar M, Manikandan M. Prevalence and antimicrobial susceptibility pattern of methicillin resistant Staphylococcus aureus: A multicentre study.Indian J Med Microbiol 2006; 24:34-38.

15. Aleksandra AD, Misic MS, Mira ZV, et al. Prevalence of inducible clindamycin resistance among community associated staphylococcal isolates in central Serbia. Indian J Med Microbiol 2014; 32:49-52.

16. Shenoy MS, Bhat GK, Kishore A, Hassan MK. Significance of MRSA strains in community associated skin and soft tissue infections. Indian $\mathrm{J}$ Med Microbiol 2010; 28:152-154. 